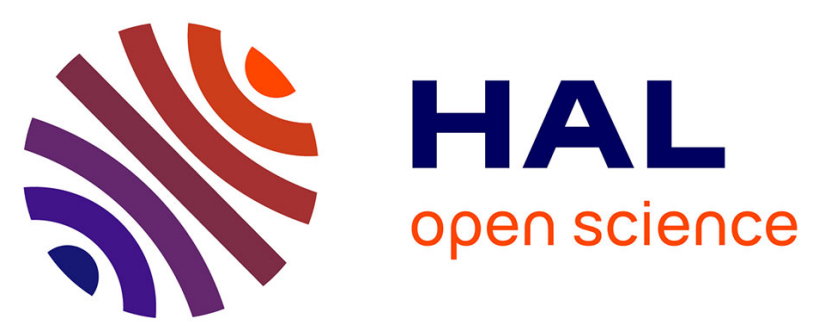

\title{
Time-resolved spectroscopy on epitaxial graphene in the infrared spectral range: relaxation dynamics and saturation behavior
}

Stephan Winnerl, F. Göttfert, Martin Mittendorff, Henri Schneider, Manfred Helm, Torben Winzer, Ermin Malic, Andreas Knorr, Milan Orlita, Marek Potemski, et al.

\section{To cite this version:}

Stephan Winnerl, F. Göttfert, Martin Mittendorff, Henri Schneider, Manfred Helm, et al.. Timeresolved spectroscopy on epitaxial graphene in the infrared spectral range: relaxation dynamics and saturation behavior. Journal of Physics: Condensed Matter, 2013, 25 (5), pp.054202. 10.1088/09538984/25/5/054202 . hal-01003137

\section{HAL Id: hal-01003137 https://hal.science/hal-01003137}

Submitted on 20 Jun 2014

HAL is a multi-disciplinary open access archive for the deposit and dissemination of scientific research documents, whether they are published or not. The documents may come from teaching and research institutions in France or abroad, or from public or private research centers.
L'archive ouverte pluridisciplinaire HAL, est destinée au dépôt et à la diffusion de documents scientifiques de niveau recherche, publiés ou non, émanant des établissements d'enseignement et de recherche français ou étrangers, des laboratoires publics ou privés. 


\title{
Time-resolved spectroscopy on epitaxial graphene in the infrared spectral range: relaxation dynamics and saturation behavior
}

\author{
S Winnerl ${ }^{1}$, F Göttfert ${ }^{1}$, M Mittendorff ${ }^{1,5}$, H Schneider $^{1}$, M Helm $^{1,5}$, T Winzer ${ }^{2}$, E \\ Malic $^{2}$, A. Knorr ${ }^{2}$, M Orlita ${ }^{3,6}$, M Potemski $^{3}$, M Sprinkle $^{4}$, C Berger $^{4}$, W A de \\ Heer $^{4}$ \\ ${ }^{1}$ Helmholtz-Zentrum Dresden-Rossendorf, P.O. Box 510119, 01314 Dresden, Germany \\ ${ }^{2}$ Technische Universität Berlin, Hardenbergstraße 3610623 Berlin, Germany \\ ${ }^{3}$ Grenoble High Magnetic Field Laboratory, CNRS-UJF-UPS-INSA F-38042 Grenoble Cedex 09, \\ France \\ ${ }^{4}$ Georgia Institute of Technology, Atlanta, Georgia 30332, USA \\ ${ }^{5}$ Technische Universität Dresden, 01062, Dresden, Germany \\ ${ }^{6}$ Faculty of Mathematics and Physics, Charles University, Ke Karlovu 5, 12116 Praha 2, Czech \\ Republic
}

Email: s.winnerl@hzdr.de

Abstract. We present the results of pump-probe experiments on multilayer graphene samples performed in a wide spectral range, namely from the near-infrared (photon energy $1.5 \mathrm{eV}$ ) to the terahertz (photon energy $8 \mathrm{meV}$ ) spectral range. In the near infrared, exciting carriers and probing at higher photon energies provides direct evidence for a hot carrier distribution. Furthermore spectroscopic signatures of the highly doped graphene layers at the interface to $\mathrm{SiC}$ are observed in the near-infrared range. In the mid-infrared range, the various relaxation mechanisms, in particular scattering via optical phonons and Auger-type processes, are identified by comparing the experimental results to microscopic modelling. Changes from induced transmission to induced absorption are attributed to probing above or below the Fermi edge of the graphene layers. This effect occurs for certain photon energies in the near infrared range, where it is related to highly doped graphene layers at the interface to $\mathrm{SiC}$, and in the far infrared range for the quasi-intrinsic graphene layers. In addition to the relaxation dynamics, the saturation of pump-induced bleaching of graphene is studied. Here a quadratic dependence of the saturation fluence on the pump photon energy in the infrared spectral range is revealed. 
PAC numbers: 78.67.Wj 81.05.ue 63.22.Rc 


\section{Introduction}

In graphene, a single layer of hexagonally ordered carbon atoms, electrons behave like massless Dirac ferminons. This means that the valence and conduction bands touch each other at the $K$ and $K^{\prime}$ point of the Brillouin zone and both bands exhibit linear dispersion characterized by a Fermi velocity $v_{F} \approx 10^{6} \mathrm{~m} / \mathrm{s}$ [1]. An important consequence of this band structure is a constant absorption coefficient $\alpha_{0}=e^{2} /\left(4 \varepsilon_{0} \hbar c\right) \approx 2.3 \%$ for visible and infrared photons [2,3]. This property makes graphene attractive for photonic and optoelectronic applications in a wide spectral range. During the last years many graphene-based devices such as fast detectors [4,5], saturable absorbers $[6,7]$, broadband polarizers [8], modulators and transparent electrodes [9] have been demonstrated (for reviews see Refs. 10,11). While most of these applications concern the visible and near-infrared (NIR) spectral range, a THz modulator [12], indications for THz gain under NIR excitation [13], and a THz detector concept [14] have been presented recently. In the far-infrared (FIR, terahertz) spectral range, the gapless nature of graphene is of particular importance. Furthermore this might be the most attractive spectral range for novel devices, because the existing technology based on standard semiconductors in this range is still much less mature as compared to the NIR range. With respect to the development of fast optoelectronic devices an understanding of the carrier relaxation dynamics is of key importance. To reveal the carrier dynamics, a large number of single-color and two-color pump-probe experiments have been performed on graphene. However, almost all studies involve pump photon energies of $1.5 \mathrm{eV}$ or higher [15-26]. So far only one single-single color [27] and one two-color [28] study has been published, in which excitation pulses in the mid- infrared (MIR) and FIR region have been applied. Similarly, studies on the saturation dynamics and applications as saturable absorbers have focused on the NIR spectral range $[6,7,29,30]$.

In this article we present an extensive time-resolved characterization of epitaxial graphene in the NIR, MIR and FIR spectral ranges where we investigate both the relaxation dynamics at low fluences and the saturation dynamics at elevated fluences. In the NIR spectral range we mainly discuss two-color pump probe experiments with a blue-shifted probe beam. These experiments provide direct evidence for a hot-carrier distribution and allow one to estimate the carrier temperature. In the MIR range we compare our experimental results to calculations based on the density matrix formalism. The calculations allow us to identify the predominant relaxation channels at different 
photon energies. In the FIR range a change in sign of the pump-probe signal is observed and ascribed to intraband absorption dominating over interband absorption for photon energies below twice the value of the Fermi energy. Finally the dependence of relaxation times and on the pump fluence is investigated, again covering the entire infrared spectral range.

\section{Experimental}

All our pump-probe experiments were performed in transmission geometry. The pumpinduced change in transmission of the sample was measured by monitoring the intensity of the probe beam as a function of the temporal delay between pump and probe pulses. The temporal delay was achieved by a mechanical delay stage operated in step-scan mode.

Different pulsed infrared sources were employed for covering the complete infrared spectral range. For the largest photon energy $E \approx 1.5 \mathrm{eV}$ a Ti:sapphire laser oscillator (wavelength: $750-840 \mathrm{~nm}$, pulse energy $10 \mathrm{~nJ}$, pulse duration $50 \mathrm{fs}$, repetition rate: 78 $\mathrm{MHz})$ was applied. NIR photons of lower energy (0.39 eV - $1.1 \mathrm{eV}$; wavelength $1.1 \mu \mathrm{m}-$ $3.2 \mu \mathrm{m}$ ) were generated by an optical parametric oscillator (OPO). The OPO was excited with the above mentioned Ti:sapphire laser and emitted a pulse train of the same repetition rate. In the OPO the $\chi^{(2)}$-nonlinearity of a crystal is exploited to generate a pair of photons, called signal and idler photon. Energy conservation requires that their frequencies obey the following relation:

$$
\hbar \omega_{\text {TiSa }}=\hbar \omega_{\text {signal }}+\hbar \omega_{\text {idler }} .
$$

The pulse duration of the OPO pulses was $200 \mathrm{fs}$, the pulse energy was in the $\mathrm{nJ}$ range. In our experiments the OPO served as a pulsed photon source in three configurations. Firstly, in a set of degenerate pump-probe experiments, idler photons (energy: 0.39 $0.69 \mathrm{eV}$ ) provided both the pump and probe beams. In a second (third) set of two-color experiments, graphene was pumped (probed) with signal (idler) photons and probed (pumped) with idler (signal) photons, respectively. For degenerate pump-probe experiments in the MIR and FIR range the free-electron laser FELBE delivered picosecond radiation pulses of $\mu \mathrm{J}$ pulse energy (photon energy: $55-310 \mathrm{meV}$ ) [31,32]. 
Pump and probe beams were focused on the sample with the spot size of the probe beam being slightly smaller as compared to the pump beam in order to achieve almost uniform pumping within the probed area. Typical spot sizes (full width at half maximum, FWHM) range from $70 \mu \mathrm{m}$ for NIR radiation to $600 \mu \mathrm{m}$ for the longest FIR wavelength. For most experiments the sample was mounted in an optical cryostat, which could be cooled to liquid He temperature. The NIR experiments were performed at room temperature only.

Various detectors were applied for monitoring the intensity of the probe beam, namely photodiodes based on Si and InGaAs for the NIR, liquid-nitrogen cooled mercurycadmium-telluride (MCT) detectors for the MIR, and a He-cooled extrinsic Ge:Ga photodetector or a He-cooled Si bolometer for the FIR.

In order to minimize scattered pump radiation on the detector, the polarization of pump and probe beam were set orthogonally to each other, and a polarization analyser was placed in front of the detector. For additional reduction of interference oscillations caused by residual pump radiation during the temporal overlap of both pulses, a fast varying delay stage (shaker) with small amplitude was employed in the NIR experiments. This technique results in a rapid variation of the time delay by $20 \mathrm{fs}$ in real-time periods shorter than the integration time of the step-scan data acquisition. Hence interference fringes are efficiently reduced, while the temporal resolution of the experiment is decreased only slightly.

The graphene samples studied in our experiments were grown by thermal decomposition of (0001)-oriented $\mathrm{SiC}$, i.e. on the C-terminated face of $\mathrm{SiC}[33,34]$. Even though this system contains multiple carbon layers, each layer behaves graphene-like rather than graphitic. Direct evidence for the linear dispersion and gapless nature was obtained by angle-resolved photoemission spectroscopy [35]. The lack of vertical electronic coupling of the graphene layers has been explained by the rotational stacking of the layers [36]. The graphene-like nature of our samples as well as their high crystalline quality has been verified by Raman spectroscopy [37], where a Lorentzian-shaped $2 D$ peak and no disorder-induced $D$ peak were observed (not shown). The $D$-peak corresponds to the highest optical phonon branch at the $K$-point [38]. Due momentum conservation the single phonon mode is forbidden. Further evidence for the graphene-like nature was provided by magneto-spectroscopy experiments $[39,40,41]$. The graphene layers are basically intrinsic except for the first few layers at the interface to $\mathrm{SiC}$. The interface layers are strongly doped giving rise to a Fermi 
energy of several hundred meV [20,21]. We note that multilayer graphene samples on $\mathrm{SiC}$ are especially well suited for a pump-probe study in the infrared range. The multiple layers result in large signals, hence a high signal-to-noise ratio can be achieved. Furthermore, unlike exfoliated graphene, these samples are not restricted to lateral sizes of a few $10 \mu \mathrm{m}$. This is important for the measurements in the FIR range, where the spot size of the beams is $\sim 0.5 \mathrm{~mm}$. Finally the high transparency of the SiC substrate in the infrared range is only interrupted by the reststrahlenband in the region from $100-200 \mathrm{meV}$. We investigated three samples, containing $\sim 40, \sim 50$ and $\sim 70$ layers of graphene, respectively. The relaxation dynamics was similar for all samples, only the absolute value of the pump-induced transmission scaled with the number of layers. The number of layers is routinely estimated by elipsometry. Nevertheless, the effective number of decoupled graphene layers seen in optical experiments in the infrared range is usually lower, as derived from our saturation experiments (see paragraph 4.2) and, for instance, from the strength of inter-Landau-level excitations $[42,43]$. We related this to a partial coverage of the SiC surface by graphene sheets and also to the partial AB stacking, e.g., into bilayers recently identified as a minor component in multilayer specimens with rotational stacking $[44,45]$. In addition, highly-doped interfacial graphene sheets become "invisible" when probing interband excitations at low energies. The linewidth obtained in the magneto-spectroscopy experiments ia a measure for the structural quality. For our samples the linewidth corresponds to a momentum scattering time of about $300 \mathrm{fs}$ [41].

\section{Relaxation dynamics}

\subsection{Relaxation dynamics in the infrared range: overview}

Before discussing the measured pump-probe signals, it is worthwhile to address the physical mechanisms, which are involved in the carrier relaxation in graphene. These include carrier-phonon and carrier-carrier scattering processes, which can be of intraband and interband type. Furthermore scattering is possible within one Dirac cone (intravalley scattering) as well as between the cone at the $K$ and $K^{\prime}$ point (intervalley scattering). In Fig. 1 three important relaxation channels are depicted. Electron-phonon scattering in graphene is dominated by the scattering via optical phonons, in particular by the $G$-mode at the $\Gamma$-point with $\hbar \Omega \approx 200 \mathrm{meV}$. Scattering by acoustic phonons is less efficient, since the phonon energy is much smaller and the electron-phonon coupling is weaker $[46,47,27]$. From theoretical studies as well as previous pump-probe experiments it has been concluded that the Coulomb interaction of carriers is very 
strong in graphene, hence carrier-carrier scattering is an important scattering mechanism. As one can see in Fig. 1(b), both energy and momentum conservation can be fulfilled in a carrier-carrier scattering process, if the two involved subprocesses occur along one line in $k$-space. This consequence of the linear band structure makes carriercarrier scattering very important in graphene. Many studies suggest a thermalization mainly by carrier-carrier scattering on a timescale of the order of $100 \mathrm{fs}$ and a subsequent cooling of the hot carrier distribution mainly by optical phonons $[15,26,48]$. A detailed study of the fluence-dependent contributions of carrier-carrier scattering and carrier-phonon scattering to the thermalization process is presented in the article by Winzer and Malic in this issue [49]. Apart from electron-electron scattering taking place solely in the conduction band as sketched in Fig. 1(b), there are Coulomb processes, where at least one subprocess is of interband type. In Fig. 1(c) such a process, namely impact excitation - also called inverse Auger scattering - is illustrated. In this process one conduction-band electron transfers energy and momentum to a valence-band electron and by that excites the latter into the conduction band $[50,51]$. There are two important implications of this process. Firstly the number of carriers in the conduction band is increased (carrier multiplication), secondly the temperature of the electron distribution in the conduction band is decreased. Note that Auger recombination is the inverse process of impact ionization and in equilibrium the contributions of both processes cancel each other out. However, shortly after the optical excitation Auger recombination is efficiently suppressed by Pauli blocking due to the filled states in the valence band resulting in a stronger impact excitation rate compared to the Auger recombination rate $[50,51]$.

(a)

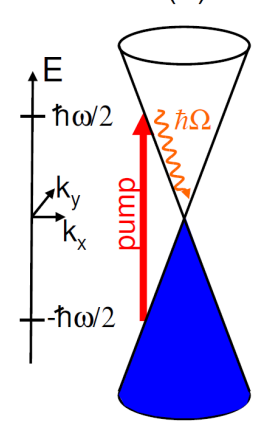

(b)

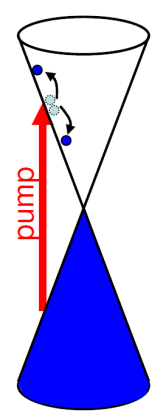

(c)

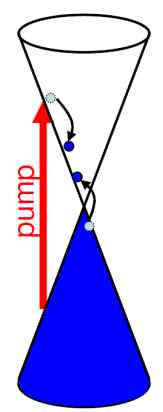


Figure 1: Schematic presentation of carrier relaxation processes in graphene after optical pumping. (a) Intraband relaxation via optical phonons with an energy $\hbar \Omega \approx 200 \mathrm{meV}$. (b) Intraband electronelectron scattering in the conduction band. (c) Impact excitation increases the number of electrons in the conduction band by bringing carriers from the valence band into the conduction band. The same illustration, but with the direction of both arrows flipped, would correspond to Auger recombination.

In Fig. 2 degenerate pump-probe signals obtained in our experiments are presented. At the highest energies ( $E=1.5 \mathrm{eV}$ and $0.55 \mathrm{eV}$ in Figs. 2(a) and 2(b)) a strong positive signal, i.e. pump induced transmission is followed by a negative signal which decays with a time constant of $\sim 2.5$ ps. The positive signal is associated with pump-induced bleaching of the transition, the negative part most likely is caused by intraband absorption. This regime will be discussed in section 4.1. Interestingly the relaxation time remains almost unchanged as the photon energy is decreased to $0.25 \mathrm{eV}$, where the signal is purely positive (cf. Fig. 2(c)). Note that the slower rise time of the signal at this energy is due to the longer pulse duration of $0.7 \mathrm{ps}$ for the FELBE pulse for $E=0.25 \mathrm{eV}$ compared to $0.2 \mathrm{ps}$ for the OPO pulse for $E=0.25 \mathrm{eV}$. As the photon energy is decreased further, a strong increase in the relaxation time is observed (cf. Fig. 1(d) - Fig. 1(g)). This slower relaxation for photon energies below the optical-phonon energy is caused by a suppression of optical-phonon scattering, which is discussed in detail in section 3.3. The pronounced increase of the relaxation time as the photon energy is decreased from 0.25 $\mathrm{eV}$ to $72 \mathrm{meV}$ is accompanied by a significant change in the temperature dependence of the pump probe signal. For $E=0.25 \mathrm{eV}$ the relaxation time for $300 \mathrm{~K}$ is only slightly shorter as compared to the case for $10 \mathrm{~K}$, while for $E=72 \mathrm{meV}$ the room temperature relaxation time is much shorter. As the photon energy is further decreased from $30 \mathrm{meV}$ to $20 \mathrm{meV}$ a change in sign of the pump-probe signal is discovered (cf. Fig. 2(e) and Fig. 2(f)). As discussed in section 3.4., this effect is related to a change from interband absorption to intraband absorption as the photon energy is decreased below twice the value of the Fermi energy.

Most of the pump-probe signals can be described well assuming a biexponential decay. The first relaxation time $\tau_{1} \approx 0.1$ ps is observed in the NIR range only. It is associated mainly with carrier-carrier scattering. A relaxation time $\tau_{2}$ in the picosecond range is present at all investigated photon energies. This time constant is related mainly to scattering via optical phonons. The dependence of this relaxation time on the photon 
energy is plotted in Fig. 3. As already mentioned above, an optical-phonon bottleneck, i.e. a strong suppression of this relaxation channel, is observed as the photon energy is decreased below the optical-phonon energy. The results presented in Fig. 3 indicate the importance of fast thermalization via Coulomb processes. If Coulomb scattering played no role and relaxation was basically determined by a cascade of optical phonon emission processes [52], one would expect a strong increase of $\tau_{2}$ for $\boldsymbol{E}<400 \mathrm{meV}$, since for these energies the intraband optical phonon emission is forbidden by energy conservation requirements (cf. Fig. 1(a)). However, no significant change of $\tau_{2}$ is observed in this energy range. This is a strong indication that Coulomb processes, which enable both intraband relaxation of hot carriers via optical phonons and interband optical phonon scattering, are essential for a realistic description of the carrier dynamics. In the FIR range we find an additional slow relaxation time constant $\tau_{3}$ that is related to scattering via acoustic phonons. We note that in our experiments $\tau_{1}$ can not be observed in the MIR and FIR ranges, because the temporal resolution, which is limited by the pulse duration, is not high enough. On the other hand, the signal components related to $\tau_{3}$ are buried in the noise in the NIR experiment, where the signal decays quickly due to the short $\tau_{2}$ time constant. 
(a)
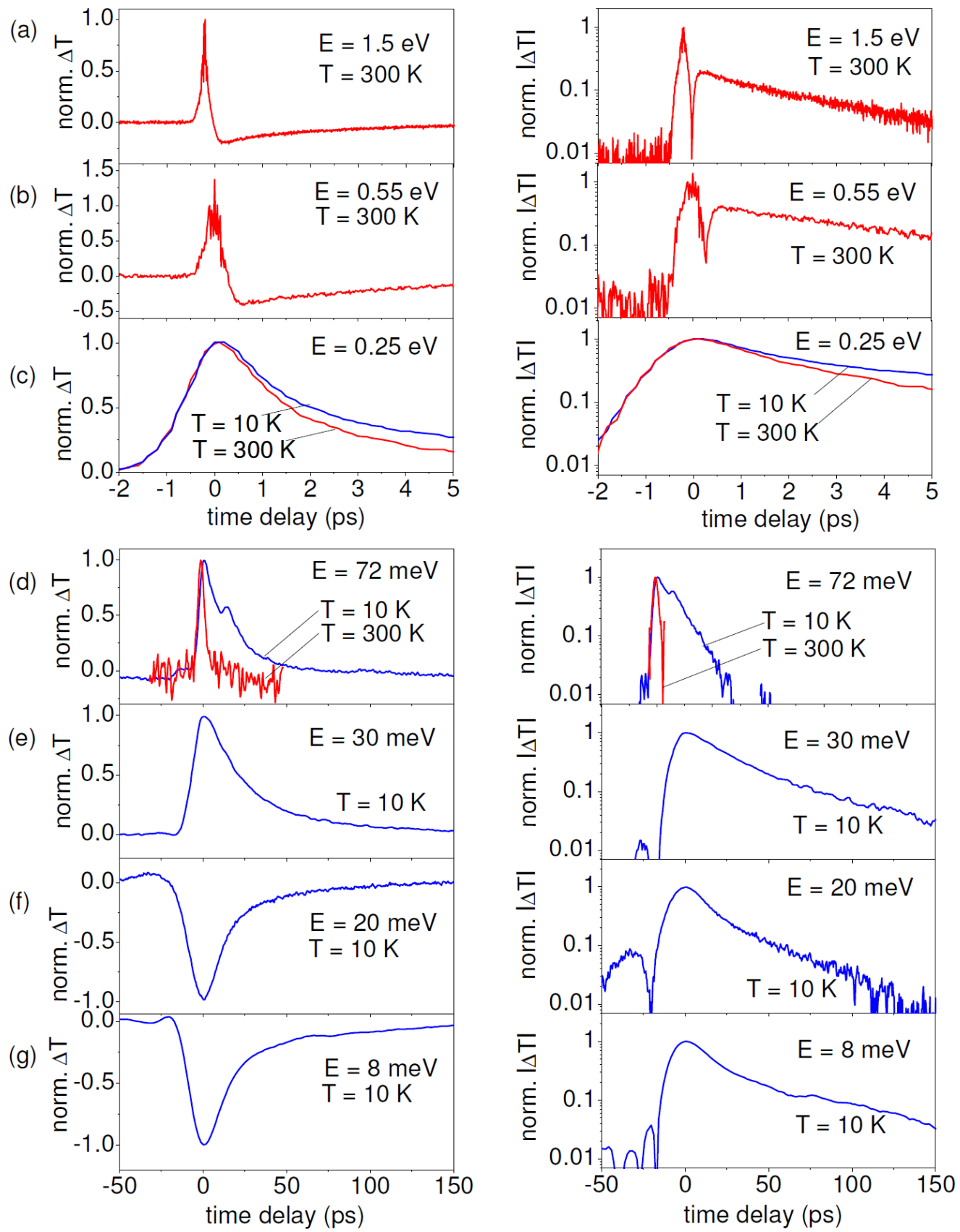

Figure 2: Normalized pump-induced transmission for different photon energies. In the left panel the curves are plotted on a linear scale, hence positive and negative contributions are visible. In the right panel the same data are plotted on a logarithmic scale, which allows one to easily compare the decay time constants. Note the different scaling of the time-delay axis for the sets (a-c) and (d-g), respectively. 


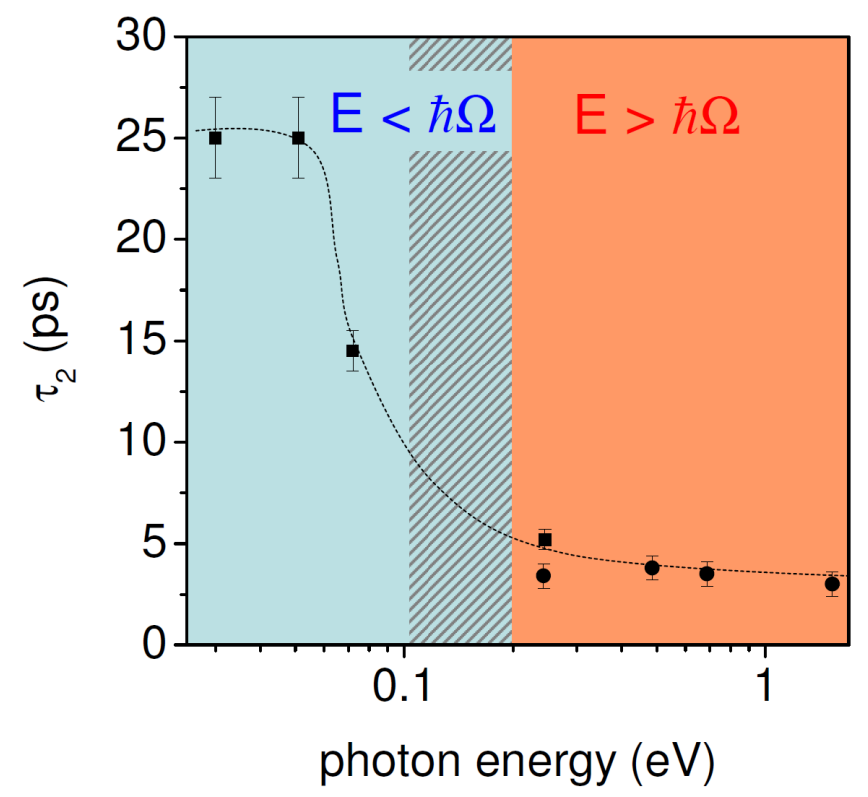

Figure 3. Dependence of the decay time $\tau_{2}$, which is mainly associated with optical-phonon scattering on photon energy. Square symbols correspond to experimental data obtained at $10 \mathrm{~K}$, round symbols to data measured at $300 \mathrm{~K}$. The dotted line is a guide for the eye. The gray shaded region indicates the reststrahlenband of $\mathrm{SiC}$, where no measurements were possible.

\subsection{Two-color NIR spectroscopy: direct evidence for a hot carrier distribution}

As mentioned in the previous section, a fast initial decay component $\tau_{1} \approx 0.1$ ps as well as the lack of an optical-phonon bottleneck around $E=400 \mathrm{meV}$ are indications for fast thermalization via carrier-carrier scattering. In the following we describe a two-color pump-probe experiment with a blue-shifted probe beam, which directly indicates that a hot carrier distribution is established on a timescale of the order of 100 fs. To this end, the idler beam of the OPO served as a pump beam, while the signal beam was applied for probing at energies above the excitation energy. Energy conservation in the OPO crystal implies that lowering the pump energy results in an increase of the probe energy (cf. Eq. 1 and Fig. 4(a)). In Fig. 4(b) pump-probe signals are shown for three probe (pump) photon energies, $0.82 \mathrm{eV}(0.68 \mathrm{eV}), 0.96 \mathrm{eV}(0.54 \mathrm{eV})$, and $1.02 \mathrm{eV}(0.48 \mathrm{eV})$. The pump fluence in all these experiments was $16 \mu \mathrm{J} / \mathrm{cm}^{2}$ and the sample ( $\sim 50$ layers $)$ was kept at room temperature. While probing at $0.82 \mathrm{eV}$ corresponds to a situation close to a degenerate pump-probe experiment, the highest probe energy exceeds the pump energy by more than a factor of two. Strong positive pump-probe signals are observed for all three combinations of pump and probe photon energies. This is direct evidence for

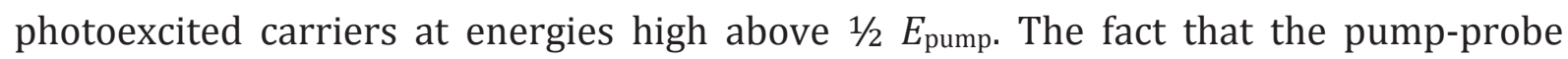


signals for different photon-energy combinations exhibit similar rise times indicates that the hot carrier distribution is formed on a timescale beyond the temporal resolution of this experiment (200 fs). Similarly, no difference in the rise time is seen by comparing blue-shifted two-color, red-shifted two-color and degenerate pump-probe signals obtained in this energy range (not shown). The maximum pump-induced transmission decreases with increasing probe energy (cf. Fig. 4 (b) and 4 (d). The pump-induced change of transmission $\Delta \mathrm{T}=\left(T-T_{0}\right) / T_{0}$, where $T$ is the transmission with optical pumping and $T_{0}$ without optical pumping, of a single layer graphene is in good approximation:

$$
\frac{\Delta T}{T_{0}} \approx-\Delta \alpha(\hbar \omega)=-\alpha_{0}\left(\Delta f_{e}\left(\frac{\hbar \omega}{2}\right)+\Delta f_{h}\left(-\frac{\hbar \omega}{2}\right)\right) .
$$

Here $\alpha_{0}=e^{2} /\left(4 \varepsilon_{0} \hbar c\right) \approx 2.3 \%$ is the universal absorption coefficient of graphene [2], $\Delta f_{e, h}(\hbar \omega, t)=f_{e, h}(\hbar \omega, t)-f_{e, h}(\hbar \omega,-\infty)$ is the difference between the distribution function of electrons or holes at a time $t$ and the equilibrium distribution function before the excitation. Assuming that after thermalization the distribution function can be approximated by Fermi functions and that the undoped layers are well described by the chemical potential set to the Dirac point, we can estimate the electron temperature from our pump-probe data. Since the experiments are performed on a multilayer system, the absorption coefficient $\alpha_{0}$ for a single layer has to be replaced by the corresponding value for the multilayer system. This value is obtained from saturating the absorption of the system (cf. section 4.2). The experimental data are best described by an electron temperature $T_{e l}=2150 \mathrm{~K} \pm 150 \mathrm{~K}$ (cf. Fig. 4(d)). 
(a)

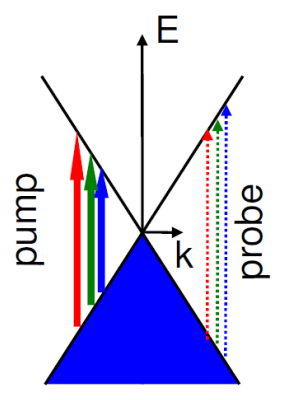

(c)

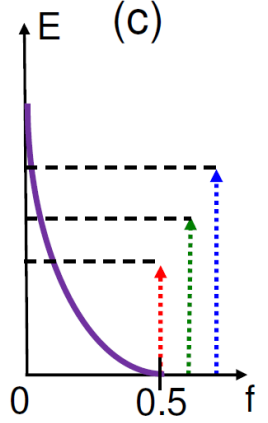

(b)

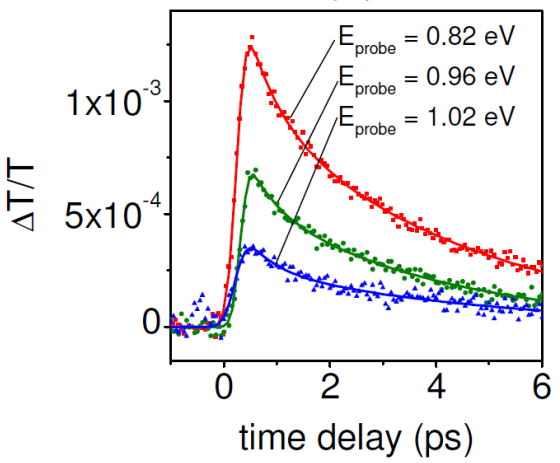

(d)

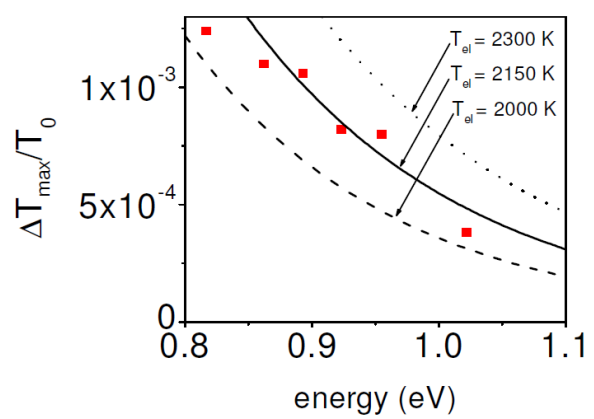

Fig. 4: Two-color pump-probe spectroscopy with a blue-sifted probe beam. (a) Schematic representation of the experiment. (b) Measured (dots) pump-probe curves, which are well described by biexponential decays (solid lines). (c) Sketch of a hot carrier distribution probed at the energies $1 / 2$ $E_{\text {probe. }}(\mathrm{d})$ Electron temperatures obtained for different probe eneries. The experimental data fit to an electron temperature of $2150 \mathrm{~K} \pm 150 \mathrm{~K}$.

Electron temperatures in the range from 1000 - $2000 \mathrm{~K}$ were deduced from degenerate pump-probe experiments with $1.5 \mathrm{eV}$ excitation energy, which investigate the same fluence range as our experiment [16]. Similarly to our experiment direct evidence for the presence of hot carriers was obtained by Obraztsov et al. [25]. In that experiment an NIR-pump - white-light probe technique was employed. In that experiment carriers at probe photon energies $\sim 30 \%$ above the pump energy could be recorded with sufficient signal-to-noise ratio. In a very recent study hot carriers were demonstrated in an extreme two-color pump-probe regime by Tani et al. [28]. Applying single-cycle THz pulses with photon energies in the meV range but high field amplitudes $(\sim 300 \mathrm{kV} / \mathrm{cm})$ carriers were excited to high energies, as confirmed by the observed induced transmission for a $1.5 \mathrm{eV}$ probe beam. In this study an interesting super-linear dependence of the induced transmission on the THz intensity was discovered. It is attributed to carrier multiplication by impact excitation [50] and seems to be the first 
clear experimental manifestation of this effect. With a technique complementary to pump-probe experiments, namely time-resolved photoluminescence, also direct evidence for hot carriers in graphene excited with NIR radiation was found [53].

\subsection{Pump-probe spectroscopy in the mid infrared: identification of an optical phonon bottleneck}

The purpose of this section is to understand the strong difference in relaxation times and in temperature dependence of the pump-probe signals obtained for $E=245 \mathrm{meV}$ and $E=72 \mathrm{meV}$ (cf. Fig. 2(c), Fig. 2(d) and Fig. 3). To this end, we compare the experimental results to microscopic theory [48,50,51].

Within the density matrix formalism [54,55], we derive the graphene Bloch-equations $[48,56]$. This set of differential equations describes the coupled dynamics of the carrier and phonon occupation together with the microscopic polarization, which is a measure for the transition probability between the conduction and valence band. We consider the carrier-light coupling as well as the Coulomb and carrier-phonon interaction in a microscopic and consistent treatment. Our approach allows time-, momentum-, and angle-resolved calculations of the optical excitation and the subsequent relaxation. To compare our microscopic results with the experiment, we approximate the differential transmission signal by the change of the carrier density $\Delta \rho_{k_{0}}$ in the state $k_{0}$ that is optically excited and probed in a degenerate pump-probe experiment. The applied equations and a detailed description of the microscopic modeling can be found in Ref. 48.

The results of the microscopic calculation resemble experimental findings well (cf. Fig. 5(a) and Fig. 5(b)). We note that some of the results of this comparison are already published in Ref. 27. In this section new calculations are presented, which further enhance the understanding of the relaxation dynamics.

In both the experiment and the theory a fast initial component on a timescale of $0.5 \mathrm{ps}$ is observed for $E=245 \mathrm{meV}$, while for $E=72 \mathrm{meV}$ the decay is characterized by the slower components only. For the temperature dependence the calculated curves also show the trend observed in the experiment, namely that the relaxation time for $E=72 \mathrm{meV}$ depends strongly on temperature while it is almost independent of temperature for $E=245 \mathrm{meV}$ (cf. Fig. (5(c)). While the qualitative trends, in particular the dependence on photon energy and temperature, agree very well between theory and experiment, there are 
quantitative differences in the relaxation times. All the experimentally observed relaxation times are roughly two times shorter as compared to the calculated times. This may be related structural defects and grain boundaries, which are contained in the graphene samples but not considered in the microscopic modeling. Further possible reasons for the somewhat longer relaxation time in the calculation compared to the experiment are discussed in Ref. 27. Comparable long relaxation times involving a strong temperature dependence have been observed in NIR-pump - THz-probe experiments [23].

For identification of the physical processes related to the decay of the pump-probe signal it is most instructive to switch off certain processes in the calculation. In Fig. 5(c) the result for the full dynamics is compared with calculations, where the optical-phonon scattering is excluded. Counterintuitively, even at this photon energy, that is significantly smaller than the optical-phonon energy, the relaxation is still dominated by scattering via optical phonons. Much longer time constants are found for the relaxation solely via acoustic phonons. The dominant nature of the optical-phonon relaxation channel is particularly pronounced at room temperature, but it is even seen at $T=10 \mathrm{~K}$. This effect is again pointing towards the strong carrier-carrier scattering. As shown in the previous section, carrier-carrier scattering leads to a hot carrier distribution involving carriers at

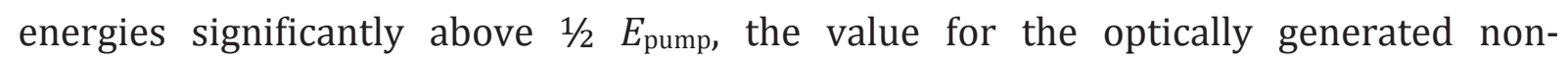
equilibrium distribution. 
(a) Experiment

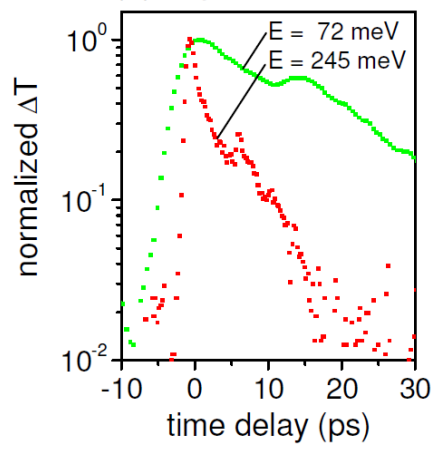

(c) Theory (b) Theory

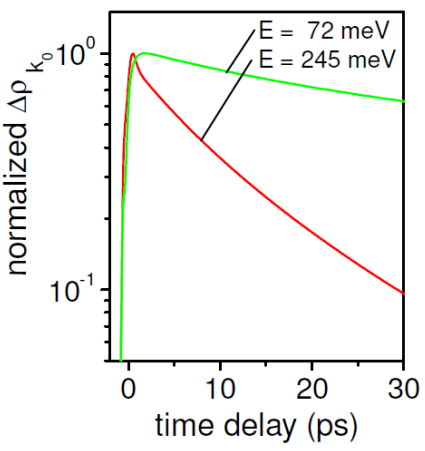

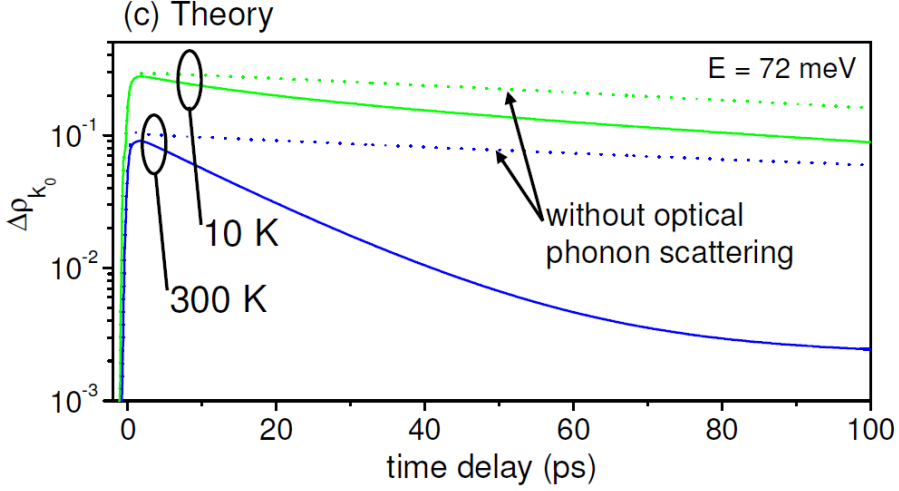

Fig 5. (a) Experimental pump-induced transmission for the photon energies $72 \mathrm{meV}$ and $245 \mathrm{meV}$ obtained at a lattice temperature of $10 \mathrm{~K}$. The secondary pulses observed at delays around 7 ps (for $E$ $=245 \mathrm{meV}$ ) and $15 \mathrm{ps}$ (for $E=72 \mathrm{meV}$ ) are experimental artifacts produced by a second pump pulse, that is caused by internal reflection in the substrate and a beamline window, respectively. (b) Calculation based on microscopic theory for the relaxation dynamics of these two photon energies for a lattice temperature of $10 \mathrm{~K}$. (c) Calculated relaxation dynamics for $10 \mathrm{~K}$ and $300 \mathrm{~K}$, taking into account the full dynamics (solid lines) and with the relaxation channel via optical phonons switched off (dotted lines). Fig. 5(b) is reprinted from Fig. 2(a) in Ref. 27.

Next we turn to the relaxation dynamics at $E=245 \mathrm{meV}$, i.e. at an energy which exceeds the optical phonon energy. We note that electrons of the non-equilibrium distribution immediately after optical excitation cannot relax via optical phonons within the conduction band, since the electron energy $1 / 2 E=123 \mathrm{meV}$ is too low. Furthermore interband relaxation via optical phonons is suppressed by Pauli blocking due to the occupied states in the valence band (cf. Fig. 6(a)). However, when Coulomb processes are included in the relaxation dynamics, two optical-phonon scattering channels open up (cf. Fig. 6(b)). One is the intraband relaxation of hot carrier within the conduction band, the other process is interband relaxation into the valence band. In order to estimate, which of the processes is dominant, microscopic modeling calculations are 
performed for the full dynamics and for the situation without interband phonon scattering. Switching off the interband phonon scattering channel results in an increase of the calculated $\tau_{2}$ from 8.3 ps to 13 ps indicating that interband phonon scattering contributes substantially (cf. Fig. 6(c)). In summary, we have observed a phonon bottleneck in the sense that optical-phonon scattering becomes less efficient for photon energies below the optical-phonon energy. However, even at lower photon energies, scattering via optical phonons is still much more efficient than scattering via acoustic phonons. For photons in the range $\hbar \Omega<E<2 \hbar \Omega$ both intraband and interband scattering via optical phonons is important.

(a)

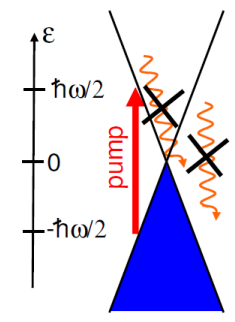

(b)

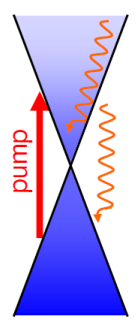

(c)

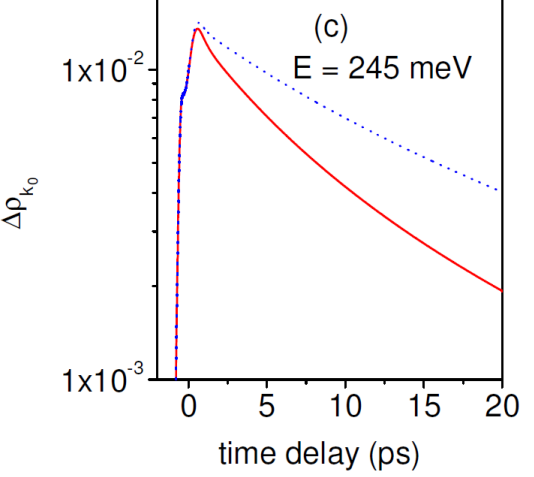

Fig. 6: Role of scattering processes for a photon energy of $245 \mathrm{meV}$. (a) Fully suppressed intraband and interband relaxation via optical phonons from the optically excited non-equilibrium carrier distribution. (b) Enabled phonon scattering of intraband and intraband type in a hot carrier distribution resulting from carrier-carrier scattering. Calculated change in the probed carrier density involving the fully dynamics (solid red line) and the dynamics without interband scattering via optical phonons (blue dotted line). The calculation is performed for a sample temperature of $10 \mathrm{~K}$

\subsection{Degenerate pump-probe spectroscopy in the far infrared: role of intraband absorption}

In this section the surprising change in sign of the pump-probe signal as the photon energy is lowered from $30 \mathrm{meV}$ to $20 \mathrm{meV}$ is explained (cf. Fig. 2(e) and Fig. 2(f)). Furthermore we discuss a second surprising effect observed in the $\mathrm{THz}$ frequency range, namely the slightly faster relaxation at lower photon energies (cf. Fig. 2(e) - (g)). While the first effect is explained already in Ref. 27, the second effect is addressed here for the first time. In order to keep the discussion self-contained, we revisit the central points of the explanation of the signreversal before we turn to the issue of the relaxation times. As depicted in Fig. 7(a), interband absorption is not possible, if the photon energy is smaller than the twice the value of the 
Fermi energy. However, free-carrier absorption results in a heating of the carrier distribution, i.e. a smearing out of the distribution around the Fermi edge. For the heated distribution interband absorption of pump photons is possible (cf. Fig. 7(a)). This means that pumping at $E<2\left|E_{f}\right|$ leads to increased absorption. In the figure the case of p-type graphene is displayed. Pump-induced absorption is similarly expected for n-type doping for $E<2\left|E_{f}\right|$. A quantitative description can be obtained referring to the real part of the dynamic conductivity $[57,24]$, which is proportional to the absorption,

$$
\begin{array}{r}
\operatorname{Re}(\sigma(\omega))=\frac{8 \sigma_{0} \tau k_{B} T_{e l}}{\pi \hbar} \ln \left(e^{-E_{f} / 2 k_{B} T_{e l}}+e^{E_{f} / 2 k_{B} T_{e l}}\right) \frac{1}{\omega \tau+1} \\
+\frac{\sigma_{0}}{2}\left[\tanh \left(\frac{\hbar \omega+2 E_{f}}{4 k_{B} T_{e l}}\right)+\tanh \left(\frac{\hbar \omega-2 E_{f}}{4 k_{B} T_{e l}}\right)\right] .
\end{array}
$$

Here $\sigma_{0}=e^{2} / 4 \hbar$ is the "universal value" of the dynamic conductivity [3] and $\tau$ the momentum relaxation time. The first summand on the right-hand side of Eq. (3) indicates intraband Drude absorption, the second one interband absorption. The real part of the dynamic conductivity is plotted for different electron temperatures in Fig. 7(b). The calculation is performed for $E_{f}=-13 \mathrm{meV}$ and $\tau=300 \mathrm{fs}$, where the latter parameter is deduced from the linewidth in magneto-spectroscopy experiments [41]. A finite absorption is found for all energies even for $T_{\mathrm{el}}=10 \mathrm{~K}$ as the Drude peak at low energies merges with the tail of the interband absorption. The increase (decrease) of the absorption with increasing temperature for the photon energy $20 \mathrm{meV}(30 \mathrm{meV})$ is indicated by the blue (red) arrow. To calculate the change in electron temperature induced by the pump beam, we assume the following simple relation,

$$
\frac{d T_{e l}}{d t}=\frac{P(t)}{c_{p}}-\frac{T_{e l}-T_{\text {latt }}}{\tau_{2}}
$$

where $\left.P(t)=I(t) \operatorname{Re}\left(\sigma\left(T_{\mathrm{el}}\right)\right) / \varepsilon_{0} c\right)$ is the absorbed power per unit area (I, laser intensity; $c$, speed of light), $c_{\mathrm{p}}$ the specific heat [58]. For $\tau_{2}$ we insert a value of $25 \mathrm{ps}$, according to the measured value for $E=30 \mathrm{meV}$. In turn we can calculate the change in transmission caused by the change in temperature according to Eq. (3). The results shown in Fig. 7(c) resemble the change in sign similarly to the experimental observation. A good agreement with the experiment is achieved for $\left|E_{f}\right|=13 \pm 2 \mathrm{meV}$, which is close to the value of $8 \mathrm{meV}$ obtained from magneto-spectroscopy experiments of samples grown by the same method. We note that the obtained values are averaged over probed area by the beams of spot diameters of $\sim 0.5 \mathrm{~mm}$. On smaller length scale the Fermi level may fluctuate due to charge puddles [59]. 


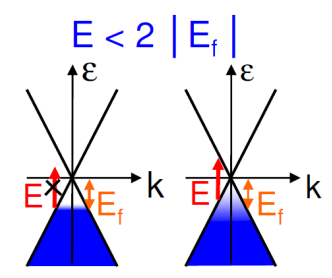

(a)
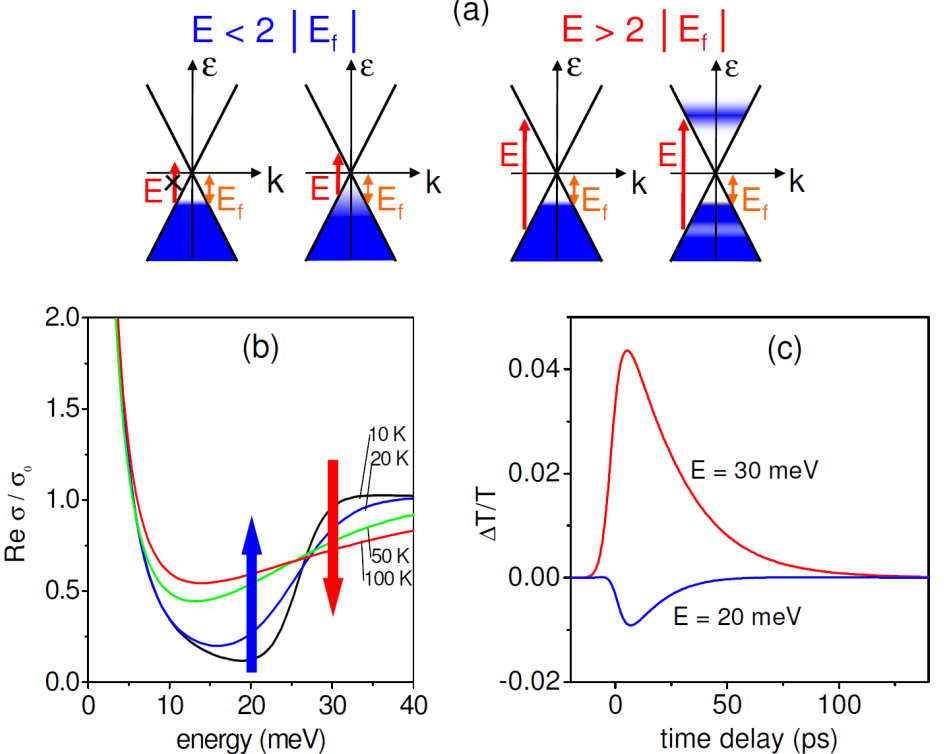

(d)

\section{induced absorption} saturable transmitter

induced transmission

saturable absorber

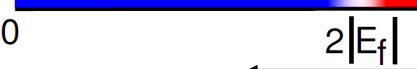

Fig. 7: (a) Pump-induced absorption due to intraband excitation increasing the electron temperature for $\mathrm{E}<2\left|E_{f}\right|$ and pump-induced induced transmission caused by interband absorption. (b) Real part of the dynamic conductivity of a single graphene layer for different temperatures $(10 \mathrm{~K}$, black; $20 \mathrm{~K}$, blue; 50 K, green; 100 K red). (c) Pump-probe signals calculated by the model described by Eq. (3) and (4) assuming a lattice temperature of $10 \mathrm{~K}$. (d) The change from induced absorption to induced transmission.

To visualize the reduced scattering time with decreasing photon energy in the THz range more clearly, the data shown in Figs. 2(e) and 2(f) are replotted in the same graph in Fig. 8(a). Interestingly, this trend is reproduced by the simple model (see Fig. 8(b)), even though the same value $\tau_{2}=25 \mathrm{ps}$ is inserted in Eq. (4) for both photon energies. This indicates that the effect may not be related to a change in the effectiveness of the underlying physical processes, in particular the scattering via optical phonons, rather it is a result of the dependence of the absorption on the electron temperature in this particular energy range. As one can see in Fig. 7(b) the change in absorption induced by a particular change in electron temperature differs significantly at the position of the two arrows. This behavior results in different decay times of the pump-probe signal associated with these particular electron temperatures. 

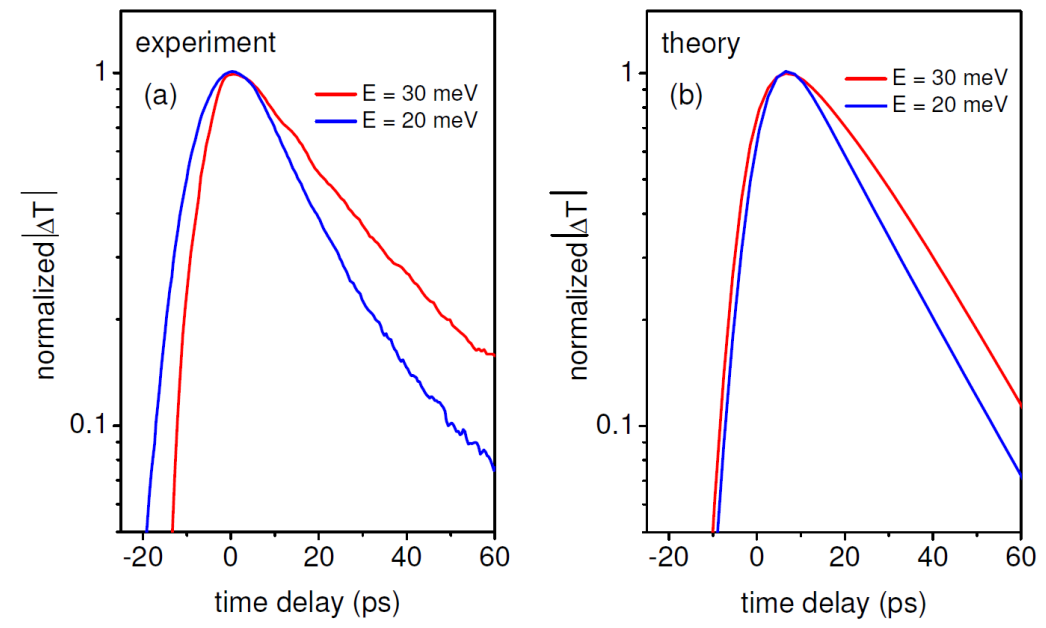

Fig. 8: Experimental (a) and calculated (b) pump-probe signals for two photon energies in the THz range. The sample is kept at $10 \mathrm{~K}$.

\subsection{Signatures of the doped interface graphene layers observed in NIR pump- probe experiments}

While the experimental results discussed previously were related to the response of the quasi-intrinsic graphene layers, we now discuss signatures arising from the highly doped graphene layers at the interface to SiC. These signatures manifest themselves as sign reversals of the pump-probe signal occurring at larger energies. In the photon energy range $0.40 \mathrm{eV}<E<0.82 \mathrm{eV}$ (wavelength range: $3.1 \mu \mathrm{m}-1.52 \mu \mathrm{m}$ ) the pumpprobe signal turns sign at specific probe energies (see table 1), where "specific" means in this context that these energies are practically independent of pump photon energy. Exemplarily this is shown in Fig. 9 for the probe (pump) energies $0.82 \mathrm{eV}(0.68 \mathrm{eV}), 0.66$ $\mathrm{eV}(0.84 \mathrm{eV}), 0.53 \mathrm{eV}(0.97 \mathrm{eV})$ and $0.51 \mathrm{eV}(0.99 \mathrm{eV})$. All results in this section were obtained at room temperature. We note that unlike all previously reported observations, which are observed reliably at all positions of the three investigated samples, this effect is seen for all samples, but strongly depends on the investigated position on each sample. Whereas at most positions the strong negative signal components are observed at the specific energies, at certain positions only small or no negative components are found. Nevertheless the effect is reproducible and observed at the same specific photon energies for all three samples. For interpreting the results we assume that the probe energy is very important for the sign changes while the pump energy does not 
significantly contribute to this effect. Since thermalization occurs on time scales below the temporal resolution of our experiment, the pump energy should be of minor importance. This is corroborated by the observation of the sign changes at the same probe energies for the case of both single-color and two-color experiments (not shown). Because the two-color signals are less obscured by interference oscillations, we concentrate on the two-color results. In Table 1 the energy ranges where negative signals tails (cf. Fig. 9(c) and (e)) are observed are listed. We associate the change from purely positive signals for $E \geq 0.82 \mathrm{eV}$ to signals with pronounced negative tails for $E \leq$ $0.66 \mathrm{eV}$ with probing above and below twice the value of the Fermi energy of the first highly doped graphene layer at the interface to SiC (cf. Fig. 9(a) and (b)). This implies that this first layer exhibits a Fermi energy $E_{f}^{(1)}=0.37 \pm 0.04 \mathrm{eV}$. Probing above the Fermi edge results in increased transmission, as the hot carriers created by the pump beam contribute to Pauli blocking. Probing below the Fermi edge, on the other hand, is accompanied by reduced transmission, since previously filled states in the conduction band are emptied, as the Fermi edge is smeared out by optical pumping. When the probe energy is decreased further, the signal switches back to a purely positive shape at a probe energy of $\sim 0.52 \mathrm{eV}$ (cf. Table 1 and Figs. 9 (e) and (f)). This is attributed to the fact that states well below the Fermi edge are not affected by the optical pumping. Hence the highly doped layer does not significantly contribute to the pump-probe signal, while the undoped layers lead to a positive pump-probe signal. Another sign change observed between the probe energies of $0.40 \mathrm{eV}$ and $0.43 \mathrm{eV}$ is associated with the probing in the vicinity of the Fermi edge of the second graphene layer, indicating $E_{f}^{(2)}=0.208 \pm 0.008 \mathrm{eV}$. Positive and negative signal components in this probe energy range were first observed by Sun et al. in two-color experiments with an amplified laser system on the same type of graphene samples as employed in our experiments [20,21]. Our values for the Fermi energies of the first two layers are in very good agreement with the values obtained by Sun et al. of $E_{f}^{(1)}=0.360 \mathrm{eV}$ and $E_{f}^{(2)}=0.215 \mathrm{eV}$ [21]. Despite of this good agreement in the transition energies for the sign reversals, the shapes of the pump-probe signals in our experiment and the ones reported by Sun et al. differ significantly. In particular the negative signal parts reported by Sun et al. are very small compared to the positive components which are present at energies in their experiment. In contrast, we observe a full reversal of the sign of the pump-probe signals when probing in the vicinity of the Fermi edges. Because of the more subtle nature of sign changes and because of the 
significantly lower signal-to-noise ratio caused by the lower repetition rate of the applied laser system, the previous experiments did not allow one to obtain relaxation times for the energy ranges associated with positive and negative signal tails, respectively. We suggest that the much larger relative strength of the negative signal components in our experiments compared to previous experiments is mainly caused by the 10 - 100 times lower fluences employed in our experiments. As we discuss in the next section, increasing of the pump fluence results in smaller negative components relative to the positive components in the NIR range. The relaxation times $\tau_{2}$ found in our experiments are somewhat larger in this energy range, where the doped layers contribute (cf. Table 1), as compared to the typical value of 2.5 - 3 ps observed for significantly higher (e.g. $1.5 \mathrm{eV}$ ) or lower (e.g. $0.25 \mathrm{eV}$ ) probe energies. We can not explain this effect, yet, nevertheless the results exclude an enhanced relaxation in the interface layers induced by coupling to substrate phonons.
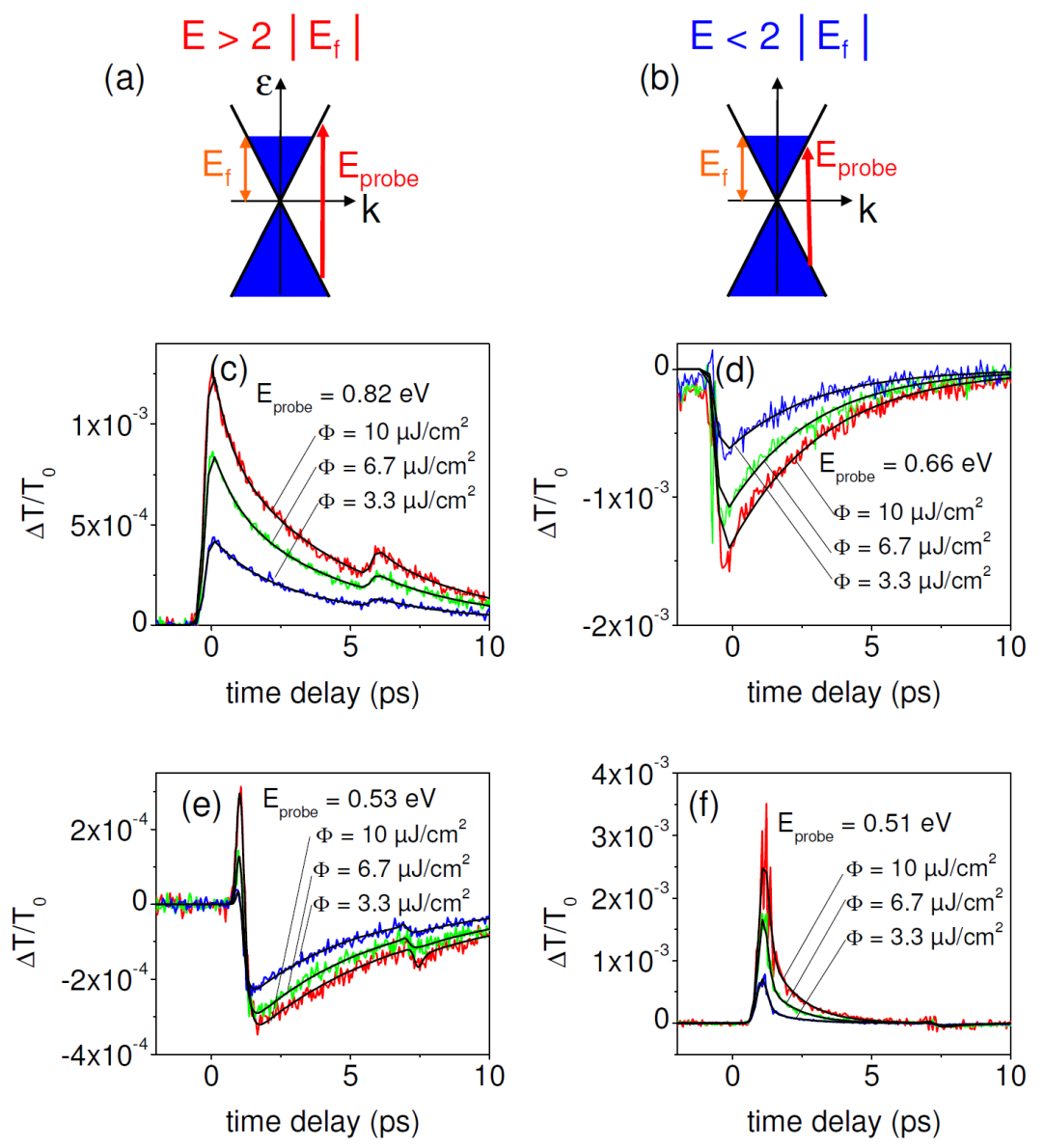

Fig. 9: Schematic illustration of probing the highly doped layer at the interface above (a) and below (b) the Fermi energy. (c-f) Two-color pump probe signals for different probe energies and pump fluences as indicated. The colored curves are experimental data, the black lines fits based on a 
biexponential decay. The steps observed in part (c), (e) and (f) around delay times of 7 ps are artifacts caused by a secondary pump pulse caused by internal reflection in the substrate. 


\begin{tabular}{|c|c|c|c|c|c|}
\hline $\begin{array}{c}\text { Photon } \\
\text { energy }\end{array}$ & $(\mathrm{eV})$ & $<0.40$ & $0.43-0.51$ & $0.53-0.66$ & $>0.82$ \\
\hline Signal tail & - & negative & positive & negative & positive \\
\hline$\tau_{2}$ & (ps) & $4 \pm 1$ & $2.5 \pm 1$ & $4 \pm 1$ & $4 \pm 1$ \\
\hline \multicolumn{5}{c}{$E_{f}^{(2)}=0.208 \pm 0.008 \mathrm{eV}$} & $E_{f}^{(1)}=0.37 \pm 0.04 \mathrm{eV}$
\end{tabular}

Table 1: Overview indicating positive and negative signal "tails" and the corresponding relaxation times $\tau_{2}$. The arrows indicate the changes in sign, which are associated with probing above and below the Fermi level $E_{f}^{(1)}$ and $E_{f}^{(2)}$ of the first and second graphene layer at the interface to SiC, respectively.

It may seem surprising that the negative signal caused by a single doped graphene layer can dominate at certain probe energies over the signal of $\sim 50$ quasi-intrinsic graphene layers. It can be understood, however, by considering that a certain change in electron temperature results in a much larger change in transmission slightly below the Fermi edge of the doped layer as compared to high above the Fermi level in case of the quasiintrinsic layers. For example, according to Eq. (3) a value $\Delta T / T_{0}=-2.5 \times 10^{-3}$ is expected for the layer with $E_{\mathrm{f}}=370 \mathrm{meV}$, while $\Delta T / T_{0}=2.1 \times 10^{-5}$ is expected for a single graphene layer with $E_{\mathrm{f}}=370 \mathrm{meV}$. Here we consider $E=0.66 \mathrm{eV}$, i.e. probing slightly below the Fermi edge of the doped layer. For this estimate an increase in electron temperature from $300 \mathrm{~K}$ to $500 \mathrm{~K}$ induced by the pump beam is assumed.

The fact that similar values for the Fermi energies of the interface layers are found reproducibly in different graphene samples on the C-terminated side of $\mathrm{SiC}$ indicates that the underlying charge transfer is of very robust nature and not affected by slightly different growth parameters. On the other hand, as mentioned previously, we find a strong lateral inhomogeneity of the samples with respect to the appearance of the negative signal tails. Further studies are necessary for understanding the reasons for this inhomogeneous structure of the interface layers. Such studies could combine pumpprobe experiments revealing the Fermi edge with techniques sensitive to structural properties such as Raman scattering. 


\section{Fluence dependence of relaxation dynamics and saturation behavior}

\subsection{Fluence dependent relaxation dynamics}

In this section we present results on the dependence of pump-probe signals on the pump fluence. The results for exitation with $1.5 \mathrm{eV}$ radiation from a Ti:sapphire laser are presented in Fig. 10. A strong positive signal, which contains some interference oscillations, is followed by a small negative signal. The measured traces are well described by the following relation, which corresponds to pumping with a Gaussian pulse and a biexponential decay:

$$
\begin{gathered}
\frac{\Delta T}{T_{0}}=A \cdot \exp \left(-\frac{t-t_{0}}{\tau_{1}}\right) \cdot\left[1-\operatorname{erf}\left(\frac{t-t_{0}}{\sqrt{2} \sigma_{s i g}}\right)+\frac{\sigma_{s i g}}{\sqrt{2} \tau_{1}}\right]+ \\
B \cdot \exp \left(-\frac{t-t_{0}}{\tau_{2}}\right) \cdot\left[1-\operatorname{erf}\left(\frac{t-t_{0}}{\sqrt{2} \sigma_{\text {sig }}}\right)+\frac{\sigma_{\text {sig }}}{\sqrt{2} \tau_{2}}\right] .
\end{gathered}
$$

The parameter $\sigma_{\text {sig, }}$, which characterizes the rise time of the pump-probe signal, is connected to the duration (full width at half maximum) of both the pump and probe pulses by the relation $\mathrm{FWHM}_{\text {pulse }}=2(\ln 2)^{0.5} \cdot \sigma_{\text {sig. }}$. Here Gaussian pulses are assumed. For all measured pump fluences $A$ is positive, while $B$ is negative. Almost independently of fluence $\tau_{1}=100 \mathrm{fs}$ is found. The decay time of the negative component, on the other hand, increases with fluences as depicted in Fig. 9(b). The amplitude of the positive component increases linearly with pump fluence in the investigated range, while $B$ exhibits a non-monotonic fluence dependence. Since both pump and probe energies here are high above the Fermi edge even of the highly doped layer at the interface, the negative component cannot be understood by the mechanism described in the previous section.

Similar pump-probe signals containing strong positive and smaller negative components have been found in many $[17,26,60,61]$, but not all $[15,16,18]$ experiments, where probing around $1.5 \mathrm{eV}$ has been applied. Unlike for the cases when the carrier distribution is probed around the Fermi edge of either intrinsic or highly doped layers, which were discussed in the last two sections, there is no widely accepted explanation for the small negative signals observed at much higher probe energies. A few possible mechanisms have been suggested to explain this effect. Malard et al. [62] have presented a model based on the dynamic conductivity including an intraband Drude term and a term for the interband transitions. They concluded that for low electron temperatures enhanced absorption dominates due to intraband contributions, while at high electron temperatures the decreased absorption due to interband transitions is the 
predominant effect. We note that this model has similarities to the one presented in section 3.4, however for a very different energy range. Another explanation, namely thermal diffusion and pump-induced shrinkage of the band separation, has been offered earlier for the small negative components observed in pump-probe experiments on graphite [63,64]. Furthermore charge transfer between the substrate and the graphene layers has been proposed [60]. The latter mechanism, however, can clearly be ruled out for the experiment of Malard et al., since that experiment was performed on freely suspended graphene. Finally Auger processes and interband recombination have been suggested as reasons for the induced absorption observed after an initial increased transmission [17]. At present, the model proposed by Malard et al. seems most convincing, since it predicts a fluence dependent change of the pump-probe signals, which agrees very well with the experimental findings described in the same paper [62]. The fluence dependencies observed in our experiment (cf. Fig. 10 (a) and (c)), which covers a much smaller fluence range as compared to the study of Malard et al. show trends that are consistent with the Malard model. We suggest that the reason for the lack of negative signal components in some of the previously reported pump-probe experiments at $E \approx 1.5 \mathrm{eV}$ is connected to the high pump-fluences employed there, e.g. $160 \mu \mathrm{J} / \mathrm{cm}^{2}$ in Ref. 15 . 

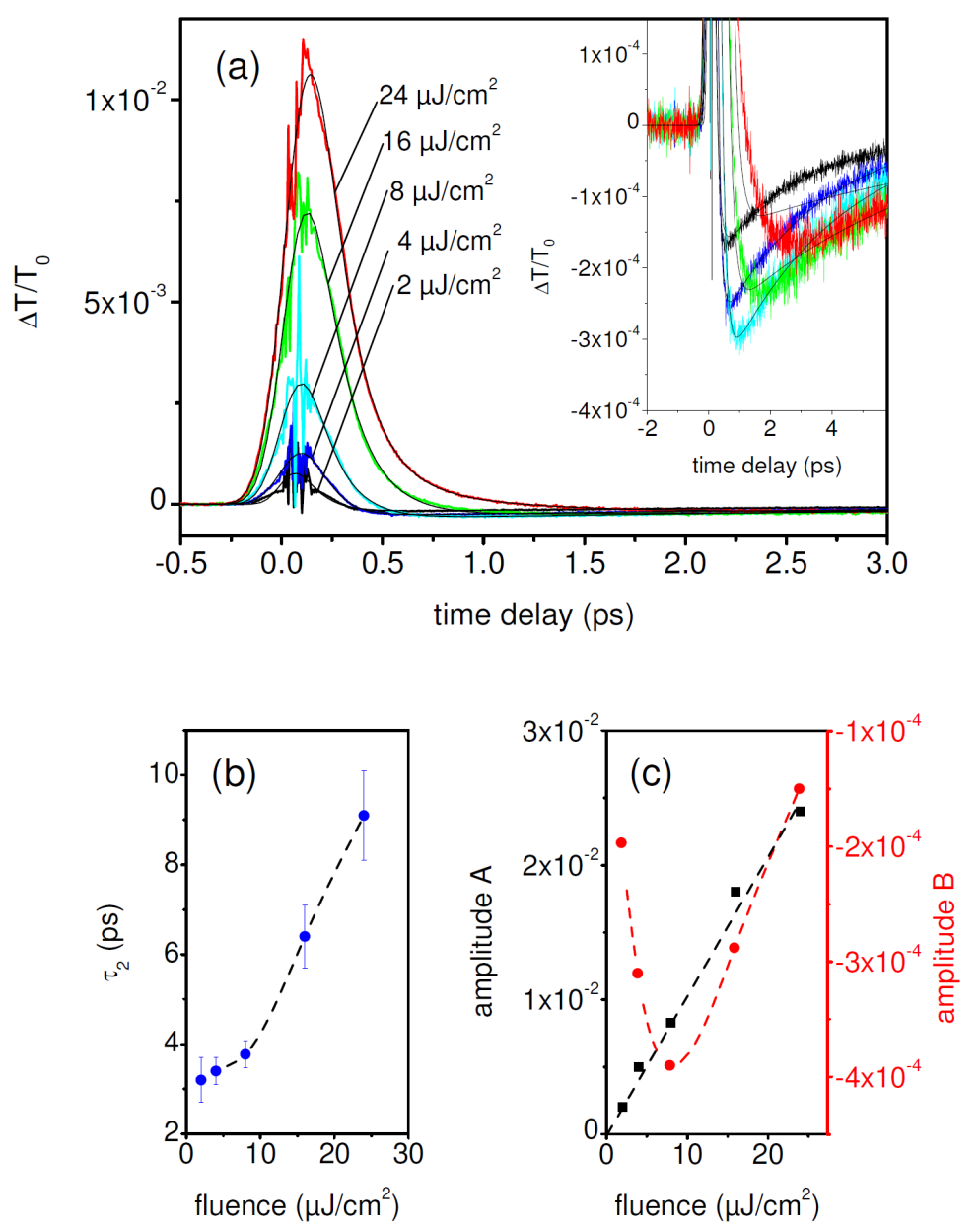

Fig. 10: (a) Single-color pump-probe signals for different pump fluences measured at for $E=1.5 \mathrm{eV}$ at room temperature. In the inset the negative parts of the signals are displayed on an enlarged vertical scale. The colored curves are experimental data, the black lines are fits according to Eq. (5). (b) Dependence of $\tau_{2}$ on pump fluence. Dependence of the amplitude A corresponding to the positive signal components and the amplitude B corresponding to the negative signal components (cf. Eq. (5)) on pump fluence. The symbols are data extracted from the measured curves, the black dashed line indicates a linear dependence, the red dashed line is a guide for the eye.

The increase of $\tau_{2}$ has also been seen in previous experiments. The effect was attributed to a hot phonon effect, i.e. the phonon temperature becoming similar to the electron temperature resulting in a slowing down of carrier relaxation [16]. An increase of the relaxation time constant is also predicted by microscopic modeling based on the density matrix formalism [49]. 
Similarly to the results obtained in the NIR range, an increase of $\tau_{2}$ with increasing pump fluence was observed for all investigated photon energies in the MIR range. Exemplarily a series of pump-probe signals for different fluences is depicted in Fig. 11. Here $\tau_{2}$ increases from 25 ps to 30 ps in the applied fluence range. Again, we attribute this effect to a decreased efficiency of the optical-phonon relaxation channel due to the presence of hot phonons. At longer delay times the slower relaxation due to scattering via acoustic phonons is visible, however here the signal-to-noise ratio for the smaller fluence values is not high enough to conclude a fluence-dependent trend of $\tau_{3}$. There is a significant difference between the fluency dependence of the pump-probe signals acquired in the MIR and NIR ranges, respectively. While in the NIR range a linear increase of the maximum induced transmission was observed for fluences up to $100 \mu \mathrm{J} / \mathrm{cm}^{2}$ (cf. Fig. $10(\mathrm{c}))$, strong saturation of the induced transmission is found for much lower fluence values in the MIR range (cf. Fig. 11). A quantitative description of this effect is the topic of the next section.

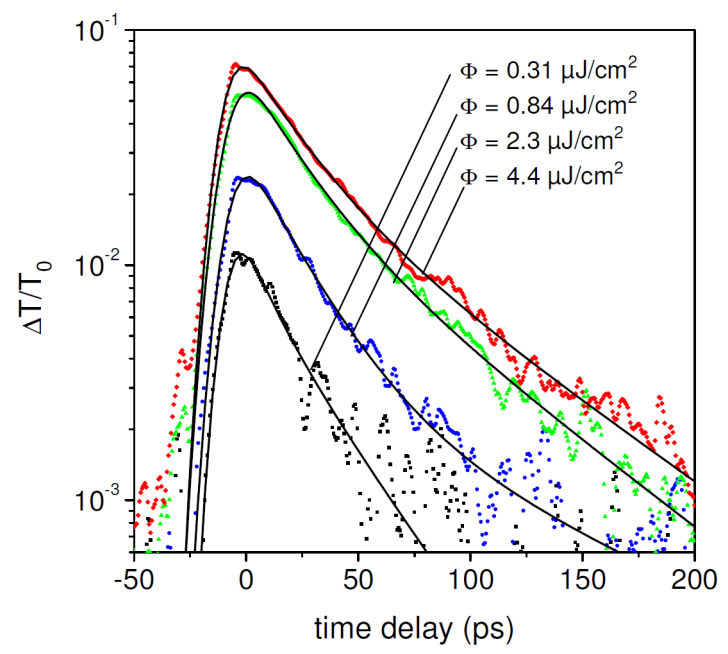

Fig. 11: Pump-probe signals for different fluences measured at $E=51 \mathrm{meV}$ and a sample temperature of $10 \mathrm{~K}$. The dots are experimental data, the lines are fits assuming a biexponential decay.

\subsection{Spectral dependence of the saturation fluence}

In the MIR range, a linear increase of the maximum induced transmission with pump fluence for low fluences is followed by a sublinear increase for higher fluences (cf. Fig. 12(a)). For all investigated photon energies the behavior can be described quantitatively by the relation 


$$
\frac{\Delta T_{\max }}{T_{0}} \propto \frac{\Phi / \Phi_{\text {sat }}}{1+\Phi / \Phi_{\text {sat }}}
$$

where $\Phi$ and $\Phi_{\text {sat }}$ denote the fluence and the saturation fluence, respectively. Eq. (6) describes the saturation behaviour of a two level system. Because of the fast thermalization, we expect the realistic situation for graphene to differ significantly from a simple two-level description, which takes into account only the energy states $-1 / 2 E$ and $1 / 2 E$. In order to estimate how significant the situation in graphene differs from such an ideal two-level situation, we estimate the number of excited carriers and compare it to the number of available states in the conduction band. Assuming a $1 \mu \mathrm{J} / \mathrm{cm}^{2}$ pulse of $E=$ $245 \mathrm{meV}$ and taking into account the $2.3 \%$ absorption of a single layer of graphene, we obtain $5.9 \times 10^{11} \mathrm{~cm}^{-2}$ excited carriers. On the other hand, the number of states in the conduction band in a $1 \%$ wide region around the energy $1 / 2 E$ is only $2.2 \times 10^{10} \mathrm{~cm}^{-2}$. The $1 \%$ wide interval resembles the region, where direct excitation by the infrared pulse of $1 \%$ spectral width is expected. For the calculation of the number of states the density of states given by

$$
\rho(\varepsilon)=g_{\nu} g_{s} \frac{1}{2 \pi \hbar^{2}} \cdot \frac{\varepsilon}{v_{F}^{2}},
$$

is employed, where $\varepsilon$ is the electron energy, $g_{\mathrm{v}}=g_{\mathrm{s}}=2$ are the valley and spin degeneracy factors, respectively, and $v_{\mathrm{F}} \approx 10^{6} \mathrm{~m} / \mathrm{s}$ is the Fermi velocity [65]. The fact that the number of generated electrons exceeds the available number of states even for a moderate fluence, where experimentally the signal is far from saturation, indicates that fast scattering out of the excited state at $1 / 2 E$ is of key importance. We suggest that Eq. (6) can still be applied, however, the obtained saturation fluence is much larger for the realistic "strongly leaky" two-level system as compared to the value for an ideal twolevel system. In Fig. 12(b) the dependence of the saturation fluence on the photon energy is depicted. Since our NIR fluences were too low to reach saturation, we refer to literature values for the NIR range. Interestingly the experimental data follow a quadratic dependence over a large range of photon energies. For an ideal two-level system we would expect a linear dependence, reflecting the linear density of states (cf. Eq. (7). The quadratic dependence points towards a linear increase of the scattering rate out of the $1 / 2 E$ state with increasing energy. We note that this scattering has to be efficient on the timescale of the pulse duration. Hence the involved scattering is mainly the carrier-carrier scattering on the $\tau_{1}$ timescale, which can not be resolved in the MIR 
range in our experiments. Intuitively one could expect that the carrier-carrier scattering rate could scale linearly with energy due to the available phase space. Another crude estimate, now taking account of fast redistribution of the excited carriers, is to count the total density of states in the entire Dirac cone up to $1 / 2 E$. Owing to Eq. (7), this number equals $1 / 4 E \rho(1 / 2 E)$, which is 50 times higher than the density within the $1 \%$ range assumed above and thus gets close to the density inferred from the density of absorbed photons. Moreover, since the number of states inside the Dirac cone scales quadratically with energy, it also explains the experimentally observed quadratic energy dependence. However, further microscopic modelling is required to show whether this dependence is required by a physical law or whether it is more an empirical trend.
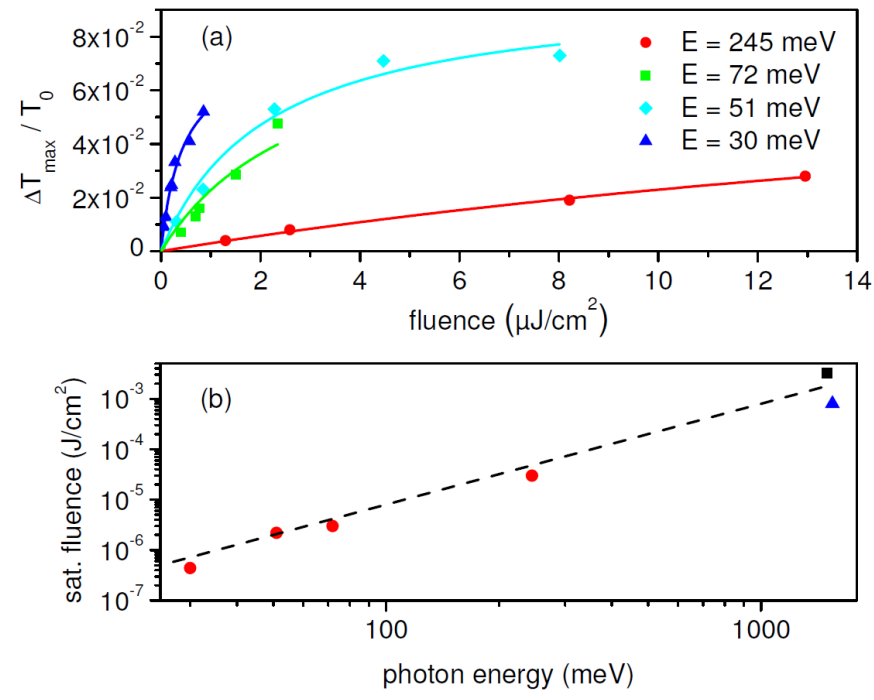

Fig. 12: (a) Saturation behaviour for different MIR photon energies. The dots represent experimental data, the lines are fits according to Eq. (6). (b) Dependence of the saturation fluence on photon energy. The red circles are our experimental data, the value indicated by black square was found by the group of Norris [66], the value indicated by the blue triangle was reported in Ref. 7. The dashed line indicates a quadratic dependence.

The study demonstrates that graphene is well suited as a saturable absorber not only in the NIR range, where many short-pulse laser applications based on graphene saturable absorbers have already been realized, but also in the MIR region. The quadratic scaling of the saturation fluence should be very useful since the laser spot area of a MIR beam is much larger compared to an NIR beam. In fact, for the same numerical aperture, the spot area scales with $E^{-2}$. The fairly long $\tau_{2}$ relaxation time of about 25 ps should not be a 
problem if the saturable absorber is used for generating short MIR pulses. Short pulse generation requires a fast rise time of the bleaching of the saturable absorber. The falling edge of the laser pulse, on the other hand, is determined by the decay of the population inversion rather than the relaxation time of the saturable absorber in case of q-switching. For modelocking also a fast rise time is sufficient to couple a large number of modes, which in turn determines the pulse duration. Hence the $\tau_{2}$ relaxation time does not limit the laser pulse duration but causes losses at too high laser. A conservative estimate for the maximum repetition rate is $f_{\text {rep }}^{\max }=\left(5 \tau_{2}\right)^{-1}=8 \mathrm{GHz}$, which is much higher than what can typically be realized in the MIR range.

Another interesting aspect of a graphene-based saturable absorber is the possibility to control its functionality by varying the Fermi energy. As discussed in sections 3.4. and 3.5., a transition from induced transmission to induced absorption can occur in graphene as the photon energy is varied across the value of twice the Fermi energy. If one can control the Fermi energy, switching from a saturable absorber material to a saturable transmitter (or optical limiter-like) material, which blocks radiation of higher intensity, is possible (cf. Fig. 7(d)). This allows for innovative device concepts such as spectral filters, which have both active (varying the Fermi energy) and passive (intensity dependent transmission change) functionality. Varying the Fermi energy by gate electrodes is widely employed for exfoliated graphene on insulating substrates. For devices in the terahertz region, however, gate-controlled graphene of larger area is required. Such a material has been demonstrated recently [67].

\section{Summary}

The relaxation dynamics in multilayer graphene samples, which are grown by thermal decomposition on the C-terminated face of $\mathrm{SiC}$, has been studied in detail in the NIR, MIR and FIR range. In the MIR range the signals are dominated by bleaching of the quasiintrinsic layers by interband absorption. In this range a strong change in the relaxation time $\tau_{2}$ is observed, which is mainly related to optical-phonon scattering. As the photon energy is decreased to values below the optical-phonon frequency, a strong increase of $\tau_{2}$ is found. While this might be expected, microscopic modelling provides additional counterintuitive insights. In particular, even though optical phonon scattering is less efficient for low photon energies, it represents still the dominant relaxation channel. The 
scattering is enabled by the presence of hot carriers at elevated energies. In the FIR range a change from pump-induced transmission to induced absorption occurs. This change in sign of the pump-probe signals is related to an interplay of interband absorption and intraband absorption in the range where the photon energy is comparable to twice the value of the Fermi energy in the quasi-intrinsic multilayers. In the NIR range, direct evidence for the hot carrier distribution is obtained in two-color experiments with a blue-shifted probe beam. Furthermore, a complex behaviour of positive and negative signals parts, which depends strongly on both the probe photon energy and the pump fluence, is revealed. The change in sign at specific NIR photon energies is attributed to probing above and below the Fermi levels of the first two highly doped graphene layers at the interface to SiC. Much smaller negative signals are also found at energies where this explanation cannot be applied. Most likely these components are related to small intraband contributions to signals that are otherwise dominated by interband absorption. In the MIR range strong saturation of the pumpprobe signals is found at elevated fluences. The saturation fluences found in these experiments as well as literature values for the NIR range scale quadratically with photon energy. This makes graphene a promising material for saturable absorbers not only for the well-studied NIR range, but also for the MIR frequency range. We expect that the main results are not limited to epitaxial graphene, however the optical properties of graphene prepared by other techniques will be modified due to different Fermi energies. The results on both the relaxation dynamics and the saturation behavior provide a solid basis for developing novel graphene-based devices, in particular for the less explored MIR and terahertz regions.

\section{Acknowledgements}

We thank M. Krause for support concerning Raman spectroscopy. Support from the German Science Foundation DFG in the framework of the Priority Program 1459 Graphene and from GACR via Grants No. P204/10/1020 and No. GRA/10/E006 is gratefully acknowledged. The research at the free-electron laser FELBE was supported by the European Community's Seventh Framework Programme (FP7/2007-2013) under Grant agreement No. 226716. We are grateful to P. Michel and the FELBE team for their dedicated support. 


\section{References}

[1] Geim A K and Novoselov K S 2007 Nature Materials 6183

[2] Nair R R, Blake P, Grigorenko A N, Novoselov K S , Booth T J, Stauber T, Peres N M R and Geim A K 2008 Science 3201308

[3] Kuzmenko A B, van Heumen E, Carbone F, and van der Marel D 2008 Phys. Rev. Lett. 100117401

[4] Xia F N, Mueller T, Lin Y M, Valdes-Garcia A and Avouris P 2009 Nature Nanotechnol. 4838

[5] Mueller T, Xia F N A and Avouris P 2010 Nature Photon. 4297

[6] Tan W D, Su C Y, Knize R J, Xie G Q, Li L J and Tang D Y, 2010 Appl. Phys. Lett. 96031106

[7] Xing G, Guo H, Zhang X, Sum T C, Huan and Cheng H A 2010 Opt. Express 184564

[8] Bao Q L, Zhang H, Wang B, Ni Z H, Lim C H Y X, Wang Y, Tang D Y and Loh K P 2011 Nature Photon. 5 411

[9] Bae S, Kim H, Lee Y, Xu X, Park J-S, Zheng Y, Balakrishnan J, Lei T, Kim H R, Song Y I, Kim Y J, Kim K S , Ozyilmaz B, Ahn J H, Hong B H and Iijima S 2010 Nature Nanotechnol. 5574

[10] Bonaccorso F, Sun Z, Hasan T and Ferrari A C 2010 Nature Photon. 4611

[11] Bao Q L and Loh K P 2012 ACS Nano 63677

[12] Sensale-Rodriguez B, Yan R, Kelly M M, Fang T, Tahy K, Hwang W S, Jena D, Liu L and H G Xing 2011 Nature Communications 3780

[13] Boubanga-Tombet S, Chan S, Watanabe T, Satou A, Ryzhii V and Otsuji T 2012 Phys. Rev. B 85, 035443

[14] Ryzhii V, Otsuji T, Ryzhii M and Shur M S 2012 J. Phys. D - Appl. Phys. 45302001

[15] Dawlaty J M, Shivaraman S, Chandrashekhar M, Rana F and Spencer M G 2008 Appl. Phys. Lett. 92 042116

[16] Wang H, Strait J H, George P A, Shivaraman S, Shields V B, Chandrashekhar M, Hwang J, Rana F, Spencer M G, Ruiz-Vargas C S and Park J 2010 Appl. Phys. Lett. 96081917

[17] Plochocka P, Kossacki P, Golnik A, Kazimierczuk T, Berger C, de Heer W A, and Potemski M, 2009 Phys. Rev. B 80245415

[18] Kumar S, Anija N, Kamaraju N, Subrahmanyam K S, Sood A K and Rao C N R 2009 Appl. Phys. Lett. 95, 191911

[19] Ruzicka B A, Werake L K, Zhao H, Wang S and Loh K P 2010 Appl. Phys. Lett. 96173106

[20] Sun D, Wu -K, Divin C, Li X, Berger C, de Heer W A, First P N and Norris T B 2008 Phys. Rev. Lett. 101 157402

[21] Sun D, Divin C, Berger C, de Heer W A, First P N and Norris T B 2010 Phys. Rev. Lett. 104136802

[22] George P A, Strait J, Dawlaty J, Shivaraman S, Chandrashekhar M, Rana F and Spencer M G 2008 Nano Lett. 84248

[23] Strait J H, Wang H, Shivaraman S, Shields V, Spencer M and Rana F Nano Lett. 114902 (2011).

[24] Choi H, Borondics F, Siegel D A, Zhou S Y, Martin M C, Lanzara A and Kaindl R A, Appl. Phys. Lett. 200994172102

[25] Obraztsov P A, Rybin M G, Tyurnina A V, Garnov S V, Obraztsova E D, Obraztsov A N and Svirko Y P 2011 Nano Lett. 111540 
[26] Breusing M, Kuehn S, Winzer T, Malic E, Milde F, Severin N, Rabe J P, Ropers C, Knorr A and Elsaesser T 2011 Phys. Rev. B 83153410

[27] Winnerl S, Orlita M, Plochocka P, Kossacki P, Potemski M, Winzer T, Malic E, Knorr A, Sprinkle M, Berger C, de Heer W A, Schneider H and Helm M 2011 Phys. Rev. Lett. 107237401

[28] Tani S, Blanchard F and Tanaka K 2012, Phys Rev. Lett. 109166603

[29] Bao Q L, Zhang H, Wang Y, Ni Z H, Yan Y L, Shen Z X, Loh K P and Tang D Y 2009 Adv. Funct. Mater. 193077

[30] Zhang H, Tang D Y, Zhao L M, Bao Q L and Loh R P 20091717630

[31] Lehnert U, Michel P, Seidel, W, Staats G, Teichert J and Wünsch R 2007 Proc. 29th International FELConference, Novosibirsk, Russia, edited by Goldenberg B, Eliseev V, Zhirkova A and Budz P (unpublished), http://accelconf.web.cern.ch/AccelConf/f07/PAPERS/MOPPH036.PDF

[32] Bhattacharyya J, Wagner M, Zybell S, Winnerl S, Stehr D, Helm M and Schneider H 2011 Rev. Sci. Instr. 82103107

[33] Berger C, Song Z, Li T, Li X, Ogbazghi A Y, Feng R, Dai Z, Marchenkov A N, Conrad A H, First P N and de Heer W A J. Phys. Chem. B 20045219912

[34] Berger C, Song Z, Li X, Wu X, Brown N, Mayou D, Li T, Hass J, Marchenkov A N, Conrad E H, First P N and de Heer W A 2006 Science 3121191

[35] Sprinkle M, Siegel D, Hu Y, Hicks J, Tejeda A, Taleb-Ibrahimi A, Le Fèvre P, Bertran F, Vizzini S, Enriquez H, Chiang S, Soukiassian P, Berger C, de Heer W A, Lanzara A and Conrad E H 2009 Phys Rev. Lett. 103226803

[36] Hass J, Varchon F, Millan-Otoya J E, Sprinkle M, Sharma N, de Heer W A, Berger C, First P N, Magaud L and Conrad E H 2008 Phys. Rev. Lett. 100, 125504

[37] Ferrari A C, Meyer J C, Scardaci V, Casiraghi C, Lazzeri M, Mauri F, Piscanec S, Jiang D, Novoselov K S, Roth S and Geim A K 2006 Phys. Rev. Lett. 97187401

[38] Maultzsch J, Reich S, Thomsen C, Requardt and Ordejón P 2004 Phys. Rev. Lett 92075501

[39] Sadowski M L, Martinez G, Potemski M, Berger C and de Heer W.A. 2006 Phys. Rev. Lett. 97266405

[40] Plochocka P, Faugeras C, Orlita M, Sadowski M L, Martinez G, Potemski M, Goerbig M O, Fuchs J N, Berger C and de Heer W A 2008 Phys. Rev. Lett. 100087401

[41] Orlita M, Faugeras C, Plochocka P, Neugebauer P, Martinez G, Maude D K, Barra A-L, Sprinkle M, Berger C, de Heer W A and Potemski M 2008 Phys. Rev. Lett. 101267601

[42] Orlita M, Faugeras C, Grill R, Wysmolek A, Strupinski W, Berger C, de Heer W A, Martinez G and Potemski M 2011 Phys. Rev. Lett. 107216603

[43] Crassee I, Levallois J, van der Marel D, Walter A L, Seyller T and Kuzmenko A B 2011 Phys. Rev. B 84 035103

[44] Siegel D A, Hwang C G, Fedorov A V and Lanzara A 2010 Phys. Rev. B 81241417

[45] Orlita M, Faugeras C, Borysiuk J, Baranowski J M, Strupinski, Sprinkle M, Berger C, de Heer W A, Basko D M, Martinez G and Potemski M 2011 Phys. Rev. B 83125302

[46] Bistritzer B and MacDonald A H 2009 Phys. Rev. Lett. 102, 206410

[47] Tse W K and Das Sarma S 2009 Phys. Rev. B 79235406

[48] Malic E, Winzer T, Bobkin E and Knorr A 2011 Phys. Rev. B 84205406 
[49] Winzer T and Malic E 2012 J Phys Cond Mat (submitted)

[50] Winzer T, Knorr A and Malic E, Nano Lett. 104839

[51] Winzer T and Malic E 2012 Phys. Rev. B 85 241404(R)

[52] Ryzhii V, Ryzhii M and Otsuji T 2007 J. Appl. Phys. 101083114

[53] Lui C H, Mak K F, Shan J and Heinz T F 2010 Phys. Rev. Lett. 105127404

[54] Lindberg M and Koch S W 1988 Phys. Rev. B 383342

[55] Axt V M and Kuhn T, 2004 Rep. Prog. Phys. 67433

[56] Stroucken T, Grönqvist J H and Koch S W 2011 Phys Rev B 84205445

[57] Mikhailov S A and Ziegler K 2007 Phys. Rev. Lett. 99016803

[58] We apply the value of $1.3 \times 10^{-6} \mathrm{~J} /\left(\mathrm{K} \mathrm{m}^{2}\right)$ in accordance with experimental and theoretical studies of graphite at $10 \mathrm{~K}$, see Nihira T and Iwata T, Phys. Rev. B 68134305

[59] Martin J, Akerman N, Ulbricht G, Lohmann T, Smet J H, von Klitzing K and Yacoby A 2007 Nature Physics 4144

[60] Newson R W, Dean J, Schmidt B and van Driel H M 2009 Opt. Express 172326

[61] Shang J, Luo Z, Cong C, Lin J, Yu T and Gurzadyan 2010 Appl. Phys. Lett. 97163103

[62] Malard L M, Mak K F, Castro Neto A H, Peres N M R and Heinz T F 2011 arXiv:1104.3104v1

[63] Seibert K, Cho G C, Kütt W, Kurz H, Reitze D H, Dadap J I, Ahn H, Downer M C and Malvezzi A M 1990 Phys. Rev. B 422842

[64] Breusing M, Ropers C and Elsaesser T 2009 Phys. Rev. Lett. 102086809

[65] Castro Neto A H, Guinea F, Peres N M R, Novoselov K S and Geim A K 2009 Rev. Mod. Phys. 81109

[66] Winzer T, Knorr A, Mittendorff M, Winnerl S, Lien M-B, Sun D, Norris T B, Helm M and Malic M 2012 Appl. Phys. Lett. (in press)

[67] Ren L, Zhang Q, Yao J, Sun Z Z, Kaneko R, Yan Z, Nanot S, Jin Z, Kawayama I, Tonouchi M, Tour J M, and Kono J 2012 Nano Lett. 123711 